\title{
Isotermas de sorção do resíduo agroindustrial bagaço de laranja
}

\author{
Leila D. Fiorentin ${ }^{1}$, Bruna T. Menon ${ }^{1}$, Sueli T. D. de Barros ${ }^{1}$, Nehemias C. Pereira ${ }^{1}, 0$ swaldo C. da M. Lima ${ }^{1} \&$ Aparecido N. Modenes ${ }^{2}$
}

\section{RESU MO}

0 bagaço de laranja úmido pode ser utilizado como adsorvente. A atividade de água é uma das variáveis que mais afetam 0 processo fermentativo e, consequentemente, 0 armazenamento deste material, razão pela qual, este trabalho teve como objetivo, a determinação das isotermas de sorção do bagaço de laranja nas temperaturas de 25,35 e $50^{\circ} \mathrm{C}$, de modo a correlacionar a umidade com a atividade de água. 0 s teores de umidade de equilíbrio foram determinados pelo método estático utilizando-se soluções de sais para uma faixa de umidade relativa de 10 a $80 \%$. Os modelos de Henderson, Chung-Pfost, Henderson Modificado, Halsey, Luikov e Motta Lima, foram ajustados aos dados experimentais, em que os resultados mostraram que as isotermas apresentaram forma sigmoidal e os modelos de Luikov e Halsey foram os que melhor representaram os dados de equilíbrio do bagaço de laranja.

Palavras-chave: resíduo de laranja, isotermas de equilíbrio, atividade de água, modelos matemáticos

\section{Sorption isotherm of agricultural residue of orange bagasse}

\begin{abstract}
Wet bagasse can be used as an adsorbent in effluent treatment process. However, before its use a previous drying is necessary. The water activity is an important parameter that affects the fermentation process, and thus its determination becomes most important in order to store this adsorbent. Thus, the objective of this work is to determine the sorption isotherms of orange bagasse at 25,35 and $50{ }^{\circ} \mathrm{C}$ temperatures in order to correlate the moisture with a water activity. The equilibrium moisture content was determined by the static method using salts solution for a $10-80 \%$ relative humidity range. The following models were fitted to the experimental data: Henderson, Chung-Pfost, M odified H enderson, Halsey, Luikov and Motta Lima. All models results followed a sigmodal-type function. The equilibrium data for the orange bagasse were better represented by the Luikov and Halsey models.
\end{abstract}

Key words: wet bagasse, equilibrium isotherms, activity of water, mathematical models 


\section{INTRODUÇÃO}

O processamento da laranja visando à obtenção do suco e dos demais subprodutos, como o óleo essencial e o D-Limoneno, gera uma quantidade elevada de resíduos sólidos denominados, industrialmente, bagaço da laranja úmido. Esses resíduos são formados, em sua maioria, pela casca (flavedo e o albedo), polpa (resíduos de vasos de suco), membranas e sementes. O flavedo é composto pela pele exterior de cor amarelada, chamada epicarpo e o albedo é a parte interior, branca, com aspecto de esponja, conhecida como mesocarpo, rico em pectina (Bampidis \& Robinson, 2006).

O resíduo da laranja pode causar muitos problemas econômicos e ambientais devido principalmente à sua elevada fermentação, pois contém cerca de $86 \%$ (b.u) de água (Tripodo et al., 2004). O bagaço pode ser utilizado como aditivo na alimentação de ruminantes na forma de ensilagem ou ração peletizada, diminuindo assim o consumo de grãos para os animais e, consequentemente, os custos de alimentação. Segundo Ítavo et al. (2000), o valor nutricional do bagaço de laranja é semelhante ao dos demais grãos utilizados na alimentação dos animais, além de apresentar elevada digestibilidade, atribuída sobretudo ao seu alto teor de carboidratos solúveis e pectina; além do uso como ração animal, a indústria tem grande interesse em desenvolver novas aplicações para o bagaço da laranja, destacando-se a utilização deste material como adsorvente no tratamento de efluentes.

A adsorção é um método empregado na separação de algumas substâncias presentes em um meio fluido, sendo o carvão ativado o adsorvente mais empregado (Corrêa et al., 2006). Vários pesquisadores têm estudado a utilização de adsorventes alternativos que apresentam, como características principais: baixo custo, elevada disponibilidade e características adsortivas semelhantes ao carvão ativado. Arami et al. (2005) e Sivaraj et al. (2001) demonstraram que a casca da laranja apresenta capacidade de remover diversos tipos de corantes têxteis presentes em efluentes, enquanto Fiorentin et al. (2006) utilizaram, como adsorvente, o bagaço da laranja úmido, na remoção de um corante têxtil, obtendo resultados semelhantes aos de Arami et al. (2005) e Sivaraj et al. (2001); entretanto, para tal aplicação é necessário realizar a secagem deste resíduo agroindustrial para estabelecer suas características adsortivas próprias e, principalmente, para inibir a ação de micro-organismos, possibilitando sua armazenagem (Camargo et al., 2007).

A secagem é a operação utilizada para evaporar a umidade contida em um material sólido, diminuindo o conteúdo líquido residual. A umidade de equilíbrio do material pode ser obtida experimentalmente e também as isotermas que relacionam o conteúdo de umidade de equilíbrio do sólido (Xe) com a umidade relativa do ar (UR) e a atividade da água $\left(a_{w}\right)$, em temperatura constante (Moura et al., 2004). A atividade de água e a umidade relativa, quando atingido o equilíbrio dinâmico, são numericamente iguais (Sousa, 2004; Kumar et al., 2005; Corrêa et al., 2006; Arslan \& Togrul, 2006; Alcântara et al., 2009). Inúmeros modelos têm sido usados para descrever matematicamente as isotermas de sorção de vários tipos de produtos alimentícios e materiais agrí- colas (Henao et al., 2009; Aviara et al., 2006; Cassini et al., 2006; Mascheroni \& Pagano, 2005; Oyelade et al., 2008; Moraga et al., 2006; Jamali et al., 2006; Sousa, 2004; Lahsasni et al., 2003; Mesquita et al., 2001) destacando-se os modelos de Henderson, Luikov, Halsey e Chung-Pfost dentre outros.

Objetivou-se com este trabalho determinar experimentalmente, pelo método estático, as isotermas de equilíbrio do bagaço de laranja nas temperaturas de 25,35 e $50{ }^{\circ} \mathrm{C}$; em seguida, ajustar os modelos de Henderson, Chung-Pfost, Henderson Modificado, Halsey, Luikov e Motta Lima aos dados experimentais e, a partir da análise estatística, avaliar o melhor ajuste.

\section{MATERIAL E MÉTODOS}

\section{Matéria-prima}

O bagaço da laranja, composto pela casca, albedo, filamentos de membrana e sementes, foi cedido por uma empresa processadora de suco de laranja, localizada no norte do Paraná, Brasil. O material, com umidade inicial de $85 \%$ (b.u.), foi acondicionado, inicialmente, em sacos de polietileno e armazenado em câmera fria a temperatura de $-10^{\circ} \mathrm{C}$ e retirado $4 \mathrm{~h}$ antes do início da operação de secagem permitindo, assim, o seu equilíbrio térmico com o meio ambiente. $\mathrm{O}$ período de acondicionamento do material variou entre 3 a 6 meses.

\section{Isotermas de equilíbrio}

A massa seca, as amostras de bagaço foram colocadas, de início, em estufa a $105^{\circ} \mathrm{C}$, por $24 \mathrm{~h}$ e, em seguida, colocadas em dessecador, por cerca de 60 min e pesadas em balança analítica (precisão $\pm 0,0001 \mathrm{~g}$ ). Deste modo, pôdese calcular a umidade de equilíbrio em base seca, Xe (b.s), pela Eq. 1.

$$
\mathrm{Xe}(\mathrm{b} . \mathrm{s})=\frac{\mathrm{M}_{\text {umi }}-\mathrm{M}_{\text {seca }}}{\mathrm{M}_{\text {seca }}}
$$

em que:

Xe (b.s) - umidade de equilíbrio, b.s

$\mathrm{M}_{\text {úmi }}$ - massa úmida, $\mathrm{g}$

$\mathrm{M}_{\text {Sec }}$ - massa seca, $\mathrm{g}$

Para obtenção dos dados de equilíbrio, utilizou-se o método estático em estufa, com o auxílio de soluções saturadas de oito tipos de sais cujas umidades relativas reais estão mostradas na Tabela 1 (Sousa, 2004).

Nos testes experimentais foram utilizados vinte e quatro frascos de vidro vedados com uma tampa de metal e fundo interno de material selante (Figura 1). Para cada um dos sais foram preparados três frascos (teste em triplicata), de modo a garantir a reprodutibilidade dos testes.

As soluções foram preparadas colocando-se $30 \mathrm{~mL}$ de água destilada dentro de cada um dos frascos, sendo que o volume final de solução variou com a quantidade de sal colocada. Acondicionaram-se os frascos com as soluções em estufa com recirculação de ar, na temperatura de $50{ }^{\circ} \mathrm{C}$, por $24 \mathrm{~h}$. 
Tabela 1. U midade relativa dos sais em função da temperatura

\begin{tabular}{ccccccccc}
\hline & \multicolumn{7}{c}{ Sal/Umidade Relativa } \\
\cline { 2 - 9 } $\mathbf{T}\left({ }^{\circ} \mathbf{C}\right)$ & $\mathrm{LiCl}$ & $\mathrm{CH}_{3} \mathbf{C O}_{2} \mathbf{K}$ & $\mathbf{M g C l}_{2}, \mathbf{6 \mathbf { H } _ { 2 } \mathbf { 0 }}$ & $\mathbf{K}_{2} \mathbf{C O}_{3}$ & $\mathbf{M g}\left(\mathbf{N O}_{3}\right)_{2}$ & $\mathbf{N a N O}_{2}$ & $\mathbf{N a C l}$ & $\mathbf{K C l}$ \\
25 & 0,113 & 0,225 & 0,328 & 0,432 & 0,511 & 0,645 & 0,753 & 0,843 \\
35 & 0,112 & 0,208 & 0,320 & 0,432 & 0,548 & 0,621 & 0,749 & 0,822 \\
50 & 0,111 & 0,192 & 0,312 & 0,433 & 0,585 & 0,597 & 0,746 & 0,802 \\
\hline
\end{tabular}

Confirmada a saturação das soluções, retirou-se os frascos de vidro da estufa para receberem as amostras de bagaço que foram retiradas do dessecador e pesadas em balança digital com precisão de 0,0001 g. Em seguida, colocou-se a amostra em um copo plástico acondicionando-a no frasco de vidro (Figura 1), o qual foi fechado e colocado na estufa em temperatura pré-estabelecida $\left(25,35\right.$ e $\left.50{ }^{\circ} \mathrm{C}\right)$.

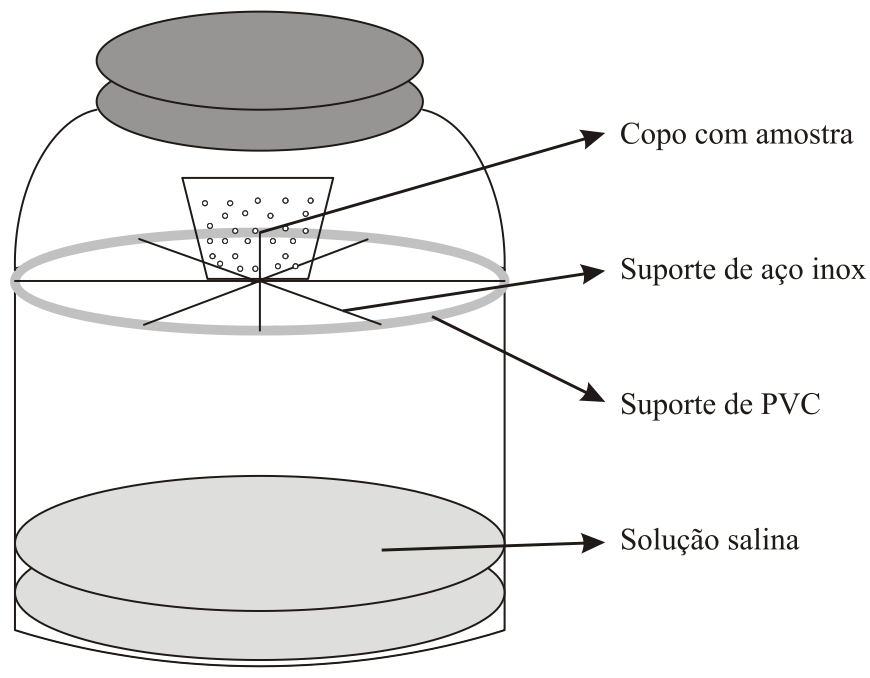

Figura 1. Frasco contendo a solução salina e a amostra de bagaço

Em intervalos de $48 \mathrm{~h}$ era realizada a pesagem do copo plástico com a amostra, procedimento este repetido até a obtenção de três leituras sucessivas constantes, o que ocorria, normalmente, ao final de 12 a 15 dias, se mantidas constantes as condições operacionais. Atingindo o equilíbrio determinaram-se as umidades das amostras (Xe) para cada sal em triplicata, usando-se o método da estufa a $105^{\circ} \mathrm{C}$, por $24 \mathrm{~h}$. Com esses resultados, obtiveram-se os pontos experimentais das isotermas de sorção, Xe em função de $\mathrm{a}_{\mathrm{w}} \mathrm{em}$ cada uma das três temperaturas testadas.

\section{Modelos matemáticos para ajuste das isotermas}

A relação entre o teor de umidade do produto analisado $(\mathrm{Xe})$, a atividade de água $\left(\mathrm{a}_{\mathrm{w}}\right)$ e a temperatura, fornece informações importantes sobre o fenômeno de sorção, tais como: a água absorvida na monocamada molecular ou o calor de sorção para as camadas subsequentes (Araújo et al., 2005).

Vários modelos têm sido propostos na literatura abordando as isotermas de sorção (Ghodake et al., 2007; Arévalo-Pinedo et al., 2006; Mohamed et al., 2005; Oliveira et al., 2005; Araújo et al., 2005). Na Tabela 2 se encontram seis modelos matemáticos com aplicação conhecida na predição de isotermas de sorção de umidade. Apesar dos mo- delos com dois parâmetros serem os mais utilizados, por facilidade de solução matemática, eles apresentam baixa precisão na determinação das isotermas de equilíbrio em amplas faixas de temperatura e umidade relativa do ar, conforme Pena et al. (2000).

Tabela 2. Modelos matemáticos de isotermas de sorção

\begin{tabular}{lcc}
\hline \multicolumn{1}{c}{ Modelo } & \multicolumn{1}{c}{ Equação } & Referência \\
Henderson & $\mathrm{Xe}=\mathrm{A}\left(\frac{1}{\mathrm{~T}} \ln \left(\frac{1}{1-\mathrm{a}_{\mathrm{w}}}\right)\right)^{\mathrm{B}}$ & $\begin{array}{c}\text { Henderson } \\
(1952)\end{array}$ \\
Chung-Pfost & $\mathrm{Xe}=\frac{-1}{\mathrm{C}} \ln \left[\left(-\frac{\mathrm{T}+\mathrm{B}}{\mathrm{A}}\right) \ln \mathrm{a}_{\mathrm{w}}\right]$ & $\begin{array}{c}\text { Chung \& Pfost } \\
(1967)\end{array}$ \\
$\begin{array}{l}\text { Henderson } \\
\text { modificado }\end{array}$ & $\mathrm{Xe}=\left[\frac{-\ln \left(1-\mathrm{a}_{\mathrm{w}}\right)}{\mathrm{A}(\mathrm{T}+\mathrm{B})}\right]^{1 / \mathrm{C}}$ & $\begin{array}{c}\text { Thompson } \\
(1972)\end{array}$ \\
Halsey & $\mathrm{Xe}=\mathrm{A}\left[\mathrm{T} \ln \left(\frac{1}{\mathrm{a}_{\mathrm{w}}}\right)\right]^{-\mathrm{B}}$ & $\begin{array}{c}\text { Iglesias \& Chirife } \\
(1976)\end{array}$ \\
Luikov & $\mathrm{Xe}=\frac{\mathrm{A}}{1+\mathrm{BT}} \ln \left(\frac{1}{\mathrm{a}_{\mathrm{w}}}\right)$ & $\begin{array}{c}\text { Keey (1992) } \\
\text { Motta Lima }\end{array}$ \\
\hline
\end{tabular}

em que:

$\mathrm{T}$ - temperatura do ar, $\mathrm{K}$

$\mathrm{a}_{\mathrm{w}}$ - atividade da água decimal, adimensional

A, B, C - constantes dos modelos que dependem da natureza do produto

As constantes dos modelos por regressão não linear (método Quasi-Newton) foram determinadas com o auxílio do software Statistica $6.0^{\circledR}$ e escolhidos os melhores modelos com base nos maiores valores do coeficiente de determinação $\left(R^{2}\right)$ e do Teste $F$ (Eq. 2) e nos menores valores da raiz do erro médio ao quadrado (RMSE), calculado pela Eq. 3 (Ertekin \& Yaldiz, 2004; Doymaz, 2005; Sousa, 2004).

$$
\begin{gathered}
\text { Teste } \mathrm{F}=\frac{\sum \overline{\mathrm{X}}_{\text {pred }}^{2}}{\sum \overline{\mathrm{X}}_{\text {resid }}^{2}} \\
\text { RMSE }=\left[\frac{1}{\mathrm{~N}} \sum_{\mathrm{i}=1}^{\mathrm{N}}\left(\mathrm{X}_{\text {exp.i }}-\mathrm{X}_{\text {pred. }}\right)^{2}\right]^{1 / 2}
\end{gathered}
$$

em que:

$\mathrm{X}_{\text {exp,i }}$ - umidade obtida experimentalmente, b.s

$\mathrm{X}_{\text {pred,i }}$ - umidade predita pelo modelo, b.s

$\mathrm{X}_{\text {resd,i }}$ - umidade residual, b.s

$\mathrm{N}$ - número de pontos experimentais 


\section{RESULTADOS E DISCUSSÃO}

As isotermas de sorção do bagaço de laranja obtidas nas temperaturas de 25,35 e $50{ }^{\circ} \mathrm{C}$ utilizando-se o método da estufa, são mostradas na Figura 2.

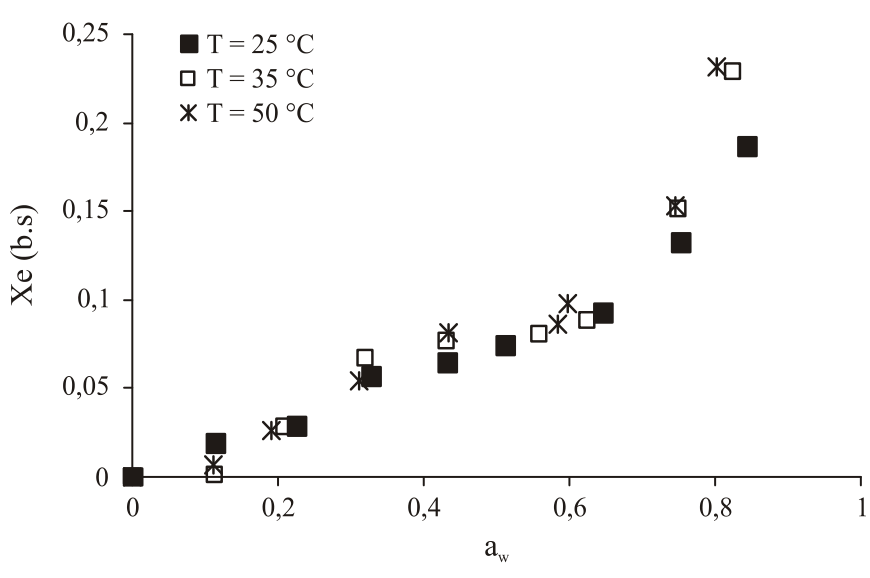

Figura 2. Isotermas de sorção do bagaço de laranja nas temperaturas de 25,35 e $50^{\circ} \mathrm{C}$

Observa-se, na Figura 2, que os dados experimentais obtidos nas temperaturas de 25,35 e $50{ }^{\circ} \mathrm{C}$ apresentaram a mesma forma sigmoidal; entretanto, para a temperatura de $35^{\circ} \mathrm{C}$ nota-se uma formação mais acentuada de 3 segmentos, sendo dois crescentes e um praticamente constante. Em relação à atividade de água $\mathrm{a}_{\mathrm{w}}$, observa-se que, para $\mathrm{a}_{\mathrm{w}}$ maior que 0,8 , a umidade de equilíbrio para a temperatura de $25^{\circ} \mathrm{C}$ foi de 0,19 , enquanto para as temperaturas de $35 \mathrm{e}$ $50{ }^{\circ} \mathrm{C}$ os valores foram aproximadamente iguais, cerca de 0,21 . Nota-se, também, que para atividades de água maiores de 0,3 , o aumento da temperatura causou um aumento da umidade de equilíbrio. Uma possível explicação para este comportamento seria a presença da casca de laranja no bagaço, cuja finalidade é a de proteger e evitar a perda de umidade do interior do fruto para o ambiente, pois, se isto não ocorresse, a laranja poderia ter uma redução elevada de umidade quando a umidade ambiente fosse baixa.

Na Figura 3 são apresentados os ajustes dos modelos de Henderson, Chung-Pfost, Henderson Modificado, Halsey, Luikov e Motta Lima, às isotermas de sorção obtidas nas temperaturas de 25,35 e $50{ }^{\circ} \mathrm{C}$.

Observa-se na Figura 3A, B e C, que para as três temperaturas analisadas os modelos de Luikov e Halsey foram os que mais se aproximaram dos dados experimentais de sorção. As isotermas obtidas apresentam comportamento característico das isotermas de equilíbrio obtidas de plantas e produtos alimentícios. Aviara et al. (2006) determinaram isotermas de dessorção do sorgo, Moraga et al. (2006) realizaram o estudo das isotermas de sorção com kiwi \& Jamali et al. (2006) estudaram a adsorção e a dessorção de folhas de Citrus reticulata obtendo formatos de curvas semelhantes.

Nas Tabelas 3, 4 e 5 são apresentados os valores das constantes dos modelos de Henderson, Chung-Pfost, Henderson Modificado, Halsey, Luikov e Motta Lima determinados com
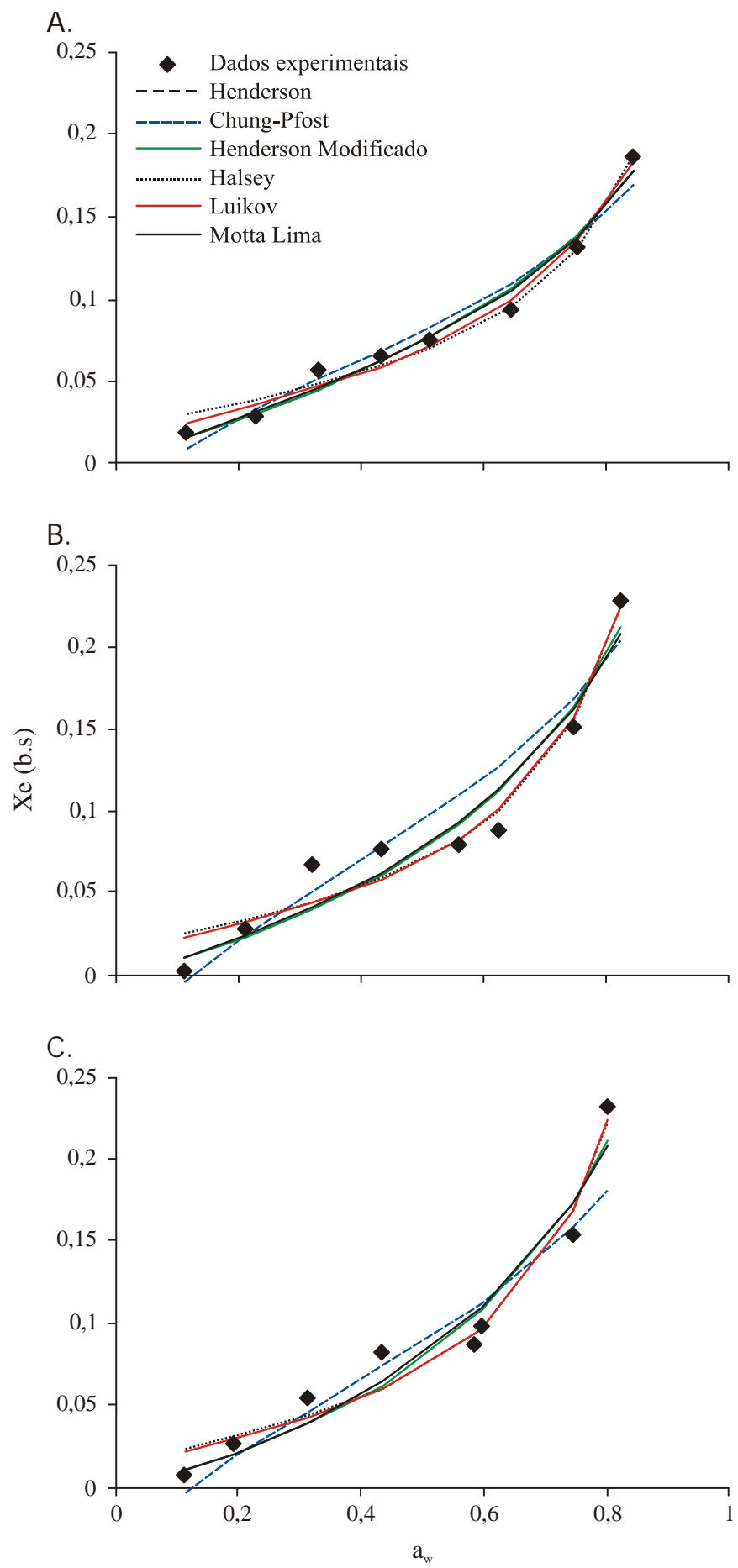

Figura 3. Isotermas de sorção do bagaço de laranja na temperatura de $25^{\circ} \mathrm{C}$ (A), temperatura de $35^{\circ} \mathrm{C}(\mathrm{B})$ e temperatura de $50^{\circ} \mathrm{C}(\mathrm{C})$, ajustadas por modelos da literatura

Tabela 3. Parâmetros de ajuste dos modelos na temperatura de $25^{\circ} \mathrm{C}$

\begin{tabular}{ccccccc}
\hline Modelo & $\mathbf{B}$ & $\mathbf{C}$ & $\mathbf{D}$ & $\mathbf{R}^{2}$ & Teste F & RMSE \\
Henderson & 0,8899 & & 8,5844 & 97,9501 & 171,534 & $7,393.10^{-3}$ \\
Chung-Pfost & $-217,781$ & 22,8191 & $-34,4697$ & 96,1084 & 89,8593 & $1,019.10^{-2}$ \\
Henderson & 0,1126 & 1,1237 & 0,0892 & 97,9510 & 171,535 & $7,393.10^{-3}$ \\
modificado & & & 3,2605 & 98,3642 & 215,205 & $6,604.10^{-3}$ \\
Halsey & 0,7236 & & 0,3985 & 98,6267 & 256,694 & $6,049.10^{-3}$ \\
Luikov & 0,0227 & & $-0,3823$ & 98,1486 & 190,054 & $7,025 \cdot 10^{-3}$ \\
Motta Lima & $-787,365$ & 0,7784 & -0, & & &
\end{tabular}


o software Statistica $6.0^{\circ}$ e os parâmetros estatísticos, utilizados para a escolha do melhor modelo.

De acordo com as Tabelas 3 a 5, os modelos de Luikov e Halsey apresentaram valores superiores de $\mathrm{R}^{2}$ e Teste $\mathrm{F}$ e inferiores de RMSE em relação aos demais modelos testados.

Tabela 4. Parâmetros de ajuste dos modelos na temperatura de $35^{\circ} \mathrm{C}$

\begin{tabular}{ccccccc}
\hline Modelo & $\mathbf{B}$ & $\mathbf{C}$ & $\mathbf{D}$ & $\mathbf{R}^{\mathbf{2}}$ & Teste F & RMSE \\
Henderson & 1,1123 & & 44,3547 & 93,7146 & 44,1992 & $1,664.10^{-2}$ \\
Chung-Pfost & $-309,229$ & 17,7852 & 17,7852 & 90,7112 & 29,5855 & $2,023.10^{-2}$ \\
Henderson & $-5,9136$ & 0,8992 & 0,0337 & 93,7141 & 44,1961 & $1,664.10^{-2}$ \\
modificado & & & 12,5441 & 95,6251 & 63,9349 & $1,388.10^{-2}$ \\
Halsey & 0,9050 & & 1,4502 & 95,5056 & 62,2229 & $1,407 \cdot 10^{-2}$ \\
Luikov & 0,06429 & & $-0,4539$ & 93,5012 & 42,7190 & $1,692 \cdot 10^{-2}$ \\
Motta Lima & $-988,5980$ & 1,1438 & & & &
\end{tabular}

Observa-se, para a temperatura de $25{ }^{\circ} \mathrm{C}$ (Tabela 3), que o modelo de Luikov foi o que apresentou os melhores valores; entretanto, para as temperaturas de 35 e $50{ }^{\circ} \mathrm{C}$ o modelo de Halsey indicou melhor desempenho que o modelo de Luikov. Sousa (2004) obteve resultados semelhantes em relação à aplicação dos modelos de Luikov e Halsey para predizer o comportamento de dados de isotermas de seda e fibras artificiais. Almeida et al. (1999) verificaram que o modelo de Halsey consegue boa representação dos dados experimentais das isotermas de gergelim. No trabalho de Ghodake et al. (2007) o modelo de Halsey Modificado teve o melhor ajuste aos dados experimentais com os menores valores de RMSE que os demais modelos testados para folhas de chá verde e chá preto, na faixa de atividade de água de 0,11 a 0,88 .

Tabela 5. Parâmetros de ajuste dos modelos na temperatura de $50^{\circ} \mathrm{C}$

\begin{tabular}{ccccccc}
\hline Modelo & $\mathbf{B}$ & $\mathbf{C}$ & $\mathbf{D}$ & $\mathbf{R}^{2}$ & Teste F & RMSE \\
Henderson & 1,1614 & & 64,5973 & 94,7102 & 53,0497 & $1,556 \cdot 10^{-2}$ \\
Chung-Pfost & $-324,89$ & 16,9512 & $-5,2534$ & 90,8223 & 30,1514 & $2,049 \cdot 10^{-2}$ \\
Henderson & 2,0570 & 0,861 & 0,02746 & 94,7098 & 53,0497 & $1,556 \cdot 10^{-2}$ \\
modificado & & & 20,963 & 96,5953 & 82,9729 & $1,248.10^{-2}$ \\
Halsey & 0,9805 & & 11,1614 & 96,5738 & 82,4604 & $1,252 \cdot 10^{-2}$ \\
Luikov & 0,9089 & & $-0,4735$ & 94,4336 & 50,3625 & $1,596 \cdot 10^{-2}$ \\
\hline Motta Lima & $-984,404$ & 1,2292 & & & &
\end{tabular}

Face aos resultados, pode-se afirmar que os modelos de Luikov e Halsey são os que melhor se aplicam para a representação dos dados de equilíbrio do bagaço de laranja.

A Figura 4 mostra os gráficos de resíduos para os respectivos modelos de Luikov e Halsey na temperatua de $25^{\circ} \mathrm{C}$ e de Halsey, nas temperaturas de 35 e $50{ }^{\circ} \mathrm{C}$.

Observa-se, na Figura 4A, B e C, uma distribuição aleatória dos resíduos, porém satisfatória nas temperaturas de 25 , 35 e $50{ }^{\circ} \mathrm{C}$; na temperatura de $25^{\circ} \mathrm{C}$, Figura $3 \mathrm{~A}$, as variações foram as menores obtidas, com valor máximo de 0,0011 e mínimo de -0,008; para as temperaturas de 35 e $50{ }^{\circ} \mathrm{C}$, Figura 3B e $\mathrm{C}$, as variações foram um pouco maiores, com valores máximos de 0,025 e mínimos de $-0,025 \mathrm{em}$ ambas as temperaturas.
A.

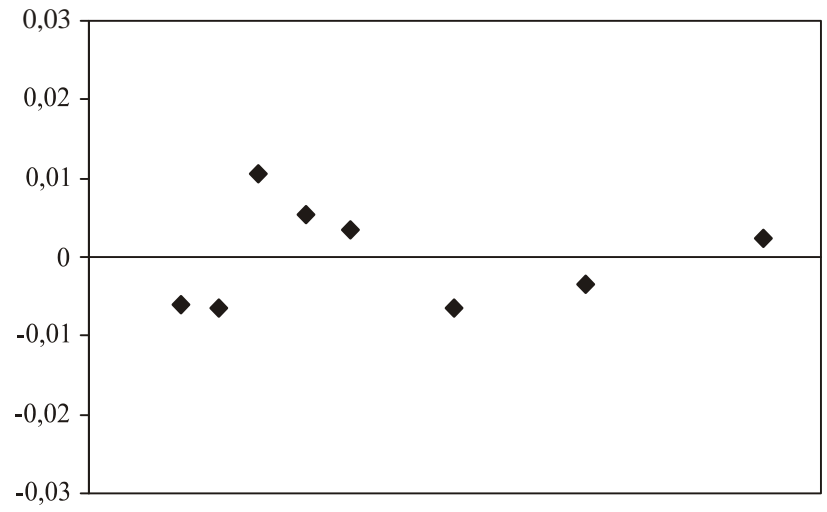

B.

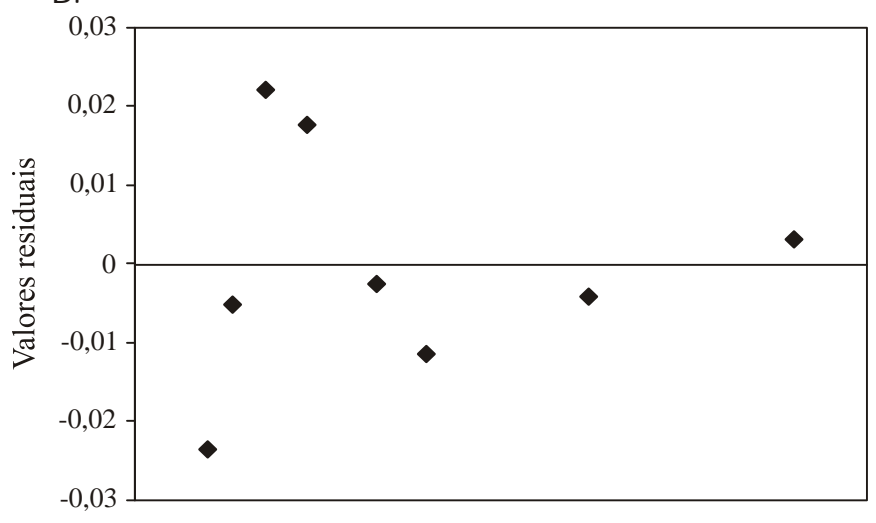

C.

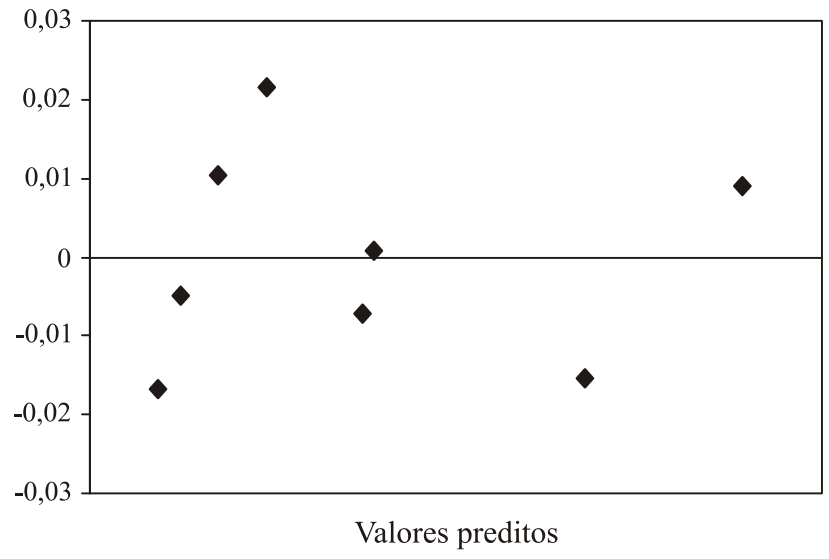

Figura 4. Resíduos estimados para o modelo de Luikov a $25^{\circ} \mathrm{C}(\mathrm{A}), 0$ modelo de $\mathrm{Halsey}$ a $35^{\circ} \mathrm{C}(\mathrm{B}), 0$ modelo de Halsey a $50^{\circ} \mathrm{C}$ (C) em função dos valores preditos

Desta maneira, os dados residuais confirmam que os modelos de Luikov e Halsey conseguem boa representação das isotermas de equilíbrio de umidade do bagaço de laranja, nas condições experimentais estudadas.

\section{CONCLUSÕES}

1. O bagaço de laranja apresenta a mesma forma sigmoidal característica das isotermas de sorção de plantas e produtos alimentícios.

2. Para a temperatura de $25^{\circ} \mathrm{C}$, o modelo de Luikov foi 
o que melhor se ajustou aos dados experimentais, enquanto para as temperaturas de 35 e $50{ }^{\circ} \mathrm{C}$ o modelo de Halsey foi o que melhor representou o comportamento dos dados de umidade de equilíbrio.

3. Para valores de atividade de água superiores a 0,20 a temperatura apresenta maior influência na umidade de equilíbrio.

\section{AGRADECIMENTOS}

Ao CNPq, pela concessão da Bolsa de Doutorado, e ao Departamento de Engenharia Química da Universidade Estadual de Maringá e da Universidade Estadual do Oeste do Paraná.

\section{LITERATURA CITADA}

Alcântara S. R.; Almeida, F. de A. C.; Silva, F. L. H. da; Gomes, J. P. Isotermas de adsorção do pedúnculo seco do caju. Revista Brasileira de Engenharia Agrícola e Ambiental, v.13, n.1, p.81-87, 2009.

Almeida, F. de A. C.; Fonseca, K. S.; Gouveia, J. P. G. de. Secagem natural de gergelim e determinação da umidade de equilíbrio. Revista Brasileira de Engenharia Agrícola e Ambiental, v.3, n.3, p.343-348, 1999.

Arami, M.; Limaee, N. Y.; Mahmoodi, N. M.; Tabrizi, N. S. Removal of dyes from colored textile wastewater by orange peel adsorbent: equilibrium and kinetic studies. Journal Colloid Interface Science, v.288, p.371-376, 2005.

Araújo, L. de F.; Oliveira, L. de. S. C.; Perazzo Neto, A.; Alsina, O. L. S. de; Silva, F. L. H. da. Equilíbrio higroscópico da palma forrageira: Relação com a umidade ótima para fermentação sólida. Revista Brasileira de Engenharia Agrícola e Ambiental, v.9, n.3, p.379-384, 2005.

Arévalo-Pinedo, A.; Santos, F. L. dos; Arévalo, Z. D. S.; Zuniga, A. D. G.; Pinedo, R. A. Desorption isotherms for murici (Byrsonima sericea) and inga (Inga edulis) pulps. Journal of Food Engineering, v.76, p.611-615, 2006.

Arslan, N.; Togrul, H. The fitting of various models to water sorption isotherms of tea stored in a chamber under controlled temperature and humidity. Journal of Stored Products Research, v.42, p.112-135, 2006.

Aviara, N. A.; Ajibola, O. O.; Aregbesola, O. A.; Adedeji, M. A. Moisture sorption isotherms of sorghum malt at 40 and $50{ }^{\circ} \mathrm{C}$. Journal of Stored Products Research, v.42, p.290-301, 2006.

Bampidis, V. A.; Robinson, P. H. Citrus by-products as ruminant feeds: A review. Animal Feed Science and Technology, v.128, p.175-217, 2006.

Camargo, G. A.; Haj-Isa, N.; Queiroz, M. R. de. Avaliação da qualidade de tomate seco em conserva. Revista Brasileira de Engenharia Agrícola e Ambiental, v.11, n.5, p.521-526, 2007.

Cassini, A. S.; Marczak, L. D. F.; Noreña, C. P. Z. Water adsorption isotherms of texturized soy protein. Journal of Food Engineering, v.77, p.194-199, 2006.
Chung, D. S.; Pfost, H. B. Adsorption and desorption of water vapour by cereal grains and their products. Part II: Development of the general isotherm equation. Transactions of the ASAE, v.10, p.549-557, 1967.

Corrêa, P. C.; A. Júnior, P. C.; Ribeiros, D. M.; Silva, F. S da. Equilíbrio higroscópico de milheto, alpiste e painço: Obtenção e modelagem. Revista Brasileira de Engenharia Agrícola e Ambiental, v.10, n.1, p.162-167, 2006.

Doymaz, I. Drying behaviour of green beans. Journal of Food Engineering, v.69, p.161-165, 2005.

Ertekin, C.; Yaldiz, O. Drying of eggplant and selection of a suitable thin layer drying model. Journal of Food Engineering, v.63, p.349-359, 2004.

Fiorentin, L. D.; Menon, B.; Alves, J. A.; Barros, S. T. D.; Santos, O. A. A.; Pereira, N. C.; Modenes, A. N. Remoção do corante reativo azul $5 \mathrm{G}$ de um efluente têxtil utilizando o bagaço de laranja como adsorvente. In: Congresso Brasileiro de Sistemas Particulados, 1, 2006, Maringá. Anais... Maringá: ENEMP, 2006. CD-Rom.

Ghodake, H. M.; Goswami, T. K.; Chakraverty, A. Moisture sorption isotherms, heat of sorption and vaporization of withered leaves, black and green tea. Journal of Food Engineering, v.78, p.827-835, 2007.

Henao, J. D.; Queiroz, M. R. de; Haj-Isa, N. M. A. Umidade de equilíbrio de café cereja descascado baseada em métodos estático e dinâmico. Revista Brasileira de Engenharia Agrícola e Ambiental, v.13, n.4, p.470-476, 2009.

Henderson, S. M. A basic concept of equilibrium moisture. Agricultural Engineering, v.33, p.20-31, 1952.

Iglesias, H.; Chirife, J. Prediction of the effect of temperature on water sorption isotherms of food material. Journal of Food Technology, v.11, p.109-116, 1976.

Ítavo, L. C. V.; Santos, G. T. dos; Jobim, C. C.; Voltolini, T. V.; Bortolassi, J. R.; Ferreira, C. C. B. Aditivos na conservação do bagaço de laranja in natura na forma de silagem. Revista Brasileira de Zootecnia, v.29, n.5, p.1474-1484, 2000.

Jamali, A.; Kouhila, M.; Mohamed, L. A.; Idlimam, A.; Lamharrar, A. Moisture adsorption-desorption isotherms of citrus reticulata leaves at three temperatures. Journal of Food Engineering, v.77, p.71-78, 2006.

Keey, R. B. Drying of loose and particulate materials, New York: Hemisphere Publication, 1992.

Kumar, A.; Singh, R. R. B.; Patil, G. R.; Pare, A. A. Effect of temperature on moisture desorption isotherms of kheer. Food Science and Technology, v.38, p.303-310, 2005.

Lahsasni, S.; Kouhila, M.; Mahrouz, M.; Fliyou, M. Moisture adsorption-desorption isotherms of prickly pear cladode (Opuntia ficus indica) at different temperatures. Energy Conversion and Management, v.44, n.1, p.923-936, 2003.

Lima, O. C. M.; Machado, G. D.; Lucheis, R. M.; Pereira, N. C. Moisture equilibrium isotherms for a handmake kraft paper. In: International Drying Symposium, São Paulo. Proceedings... São Paulo: IDS', 2004. CD-Rom.

Mascheroni, R. H.; Pagano, A. M. Sorption isotherms for maranth grains. Journal of Food Engineering, v.67, p.441-450, 2005. 
Mesquita, J. B.; Andrade, E. T. de; Corrêa, P. C. Modelos matemáticos e curvas de umidade de equilíbrio de sementes de jacarandá-da-bahia, angico-vermelho e óleo-copaíba. Cerne, v.7, n.2, p.12-21, 2001.

Mohamed, L. A.; Kouhila, M.; Jamali, A.; Lahsasni, S.; Mahrouz, M. Moisture sorption isotherms and heat of sorption of bitter orange leaves (Citrus aurantium). Journal of Food Engineering, v.67, p.491-498, 2005.

Moraga, G.; Navarrete, N. M.; Chiralt, A. Water sorption isother$\mathrm{ms}$ and phase transistions in kiwifruit. Journal of Food Engineering, v.72, p.147-156, 2006.

Moura, R. S. F. de; Gouveia, J. P. G. de; Almeida, F. de A. C.; Sousa, A. G. de; Silva, M. M. da; Oliveira, A. M. de V. Modelagem matemática para isotermas em polpa de caju. Revista de Biologia e Ciências da Terra, v.4, n.2, p.1-11, 2004.

Oliveira, M. M. de; Campos, A. R. N.; Gomes, J. P.; Silva, F. L. H. da. Isotermas de sorção do resíduo agroindustrial de casca do abacaxi (Ananas comosus L. Mer). Revista Brasileira de Engenharia Agrícola e Ambiental, v.9, n.4, p.565-569, 2005.
Oyelade, O. J.; Tunde-kintunde, T. Y.; Igbeka, J. C.; Oke, M. O.; Raji, O. Y. Modelling moisture sorption isotherms for maize flour. Journal of Stored Products Research, v.44, p.179-185, 2008.

Pena, R. S.; Ribeiro, C. C.; Grandi, J. G. Aplicação de modelos matemáticos bi- e tri-paramétricos na predição de isotermas de adsorção de umidade do guaraná (Paullinia Cupana) em pó. Ciência e Tecnologia de Alimentos, v.20, n.1, p.8-11, 2000.

Sivaraj, R.; Namasivayam, C.; Kadirlevu, K. Orange peel as an adsorbent in the removal of acid violet 17 (acid dye) from aqueous solutions. Waste Management, v.21, p.105-110, 2001. Sousa, L. H. C. D.; Perri, V. R. S.; Pereira, N. C.; Motta Lima, O. C.; Mendes, E. S. Isotermas de umidade de equilíbrio para tecidos de algodão - adsorção e dessorção. In: Congresso Brasileiro de Engenharia Química, 1, 2004, Anais... Curitiba: COBEQ, 2004. CD-Rom.

Thompson, H. L. Temporary storage of high moisture shelled corn using continuous aeration. Transactions of the ASAE, v.15, p.333-337, 1972.

Tripodo, M. M.; Lanuzza, F. Micali, G.; Coppolino, R.; Nucita, F. Citrus waste recovery: a new environmentally friendly procedure to obtain animal feed. Bioresource Technology, v.91, p.111-115, 2004. 